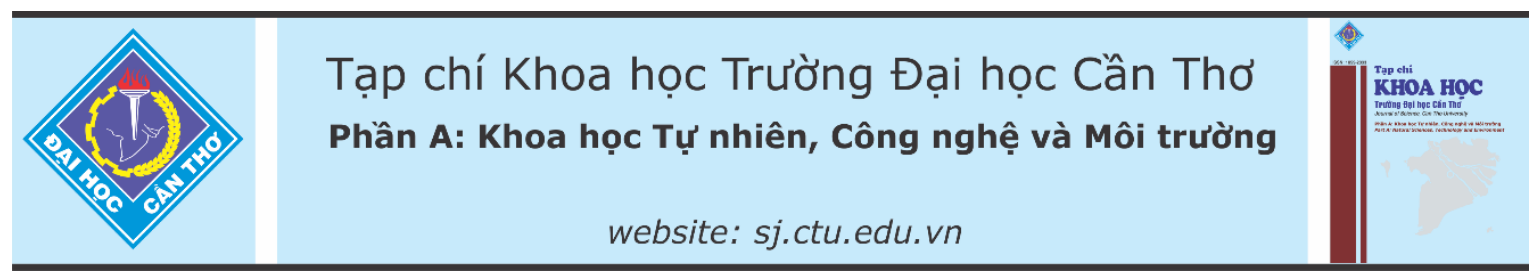

DOI:10.22144/ctu.jvn.2021.110

\title{
NHẬN DẠNG ĐỘNG CƠ KHÔNG ĐỒNG BỘ SÁU PHA SỬ DỤNG MẠNG NEURON RBF
}

\author{
Nguyễn Việt Trung* và Phạm Thanh Tùng \\ Truờng Đại học Su pham Kỹ thuật Vĩnh Long \\ *Nguoòi chịu trách nhiệm về bài viết: NguyễnViệt Trung (email: trungnv@vlute.edu.vn)
}

\section{Thông tin chung:}

Ngày nhận bài: 29/03/2021

Ngày nhận bài sưa: 20/05/2021

Ngày duyệt đăng: 20/08/2021

\section{Title:}

Identification of six phase induction motor using RBF neural network

\section{Tù khóa:}

Động co không đồng bộ sáu pha, hệ truc cố định $(\alpha \beta)$,

mang neuron $R B F$, nhận dạng hệ thống

\section{Keywords:}

Six phase induction motor, axis $(\alpha \beta)$, RBF neural network, system identification

\section{ABSTRACT}

System identification is one of the first things that must be done when solving an automatic control problem because it is impossible to analyze and synthesize the system without the mathematical model that describes the system. This paper presents and simulates the identification method of six phase induction motors using RBF (Radial Basis Function) neural network, identification parameters include speed, torque and the rotor flux on the fixed $(\alpha \beta)$ shaft system. The RBF neural network is built and trained online based on input and output data of the object. Simulation results using Matlab/Simulink software show that the error of the identification coverges to 0. Identifying parameters follow the object parameters during the engine starting time, after load and is active white noise, the match between output response and recognition response is between $98 \%-99 \%$. This study is a prerequisite to high performance control methods such as DTC (Direct Torque Control), FOC (Field Oriented Control), Fuzzy Logic be more advantageous.

\section{TÓM TẮT}

Nhận dạng hệ thống là một trong nhũng công việc đầu tiên phải thực hiện khi giải quyết bài toán điều khiển tư động bởi vì không thể phân tích, tổng hợ hệ thống khi không có mô hình toán mô tả hệ thống. Bài báo này trình bày và mô phỏng phwơng pháp nhận dạng động co không đồng bộ sáu pha sư dụng mang neuron hàm cơ sở xuyên tâm (RBF - Radial Basis Function), thông số nhận dạng gồm có tốc độ quay, mômen xoắn, tù thông rotor trên hệ trục cố định $(\alpha \beta)$. Mạng RBF được xây dưng, huấn luyện trực tuyến dựa trên dũ liệu vào ra của đối tượng. Kết quả mô phỏng dùng phần mềm Matlab/Simulink cho thấy sai số của bộ nhận dạng hội tụ về 0 . Thông số nhận dạng bám theo thông số đối tương trong khoảng thời gian động co khởi động, sau khi lắp tải và có nhiễu trắng tác động, độ phù hơpp giữa đáp ứng ngõ ra và đáp ứng nhận dạng nằm trong khoảng 98\%-99\%. Nghiên cứu này là tiền đề để tiến đến các phuơng pháp điều khiển hiệu suất cao nhu điều khiển trục tiếp mômen (DTC-Direct Torque Control), điều khiển tựa tù̀ thông (FOC - Field Oriented Control), Logic mò̀ được thuận lợi hơn. 


\section{GIỚI THIẸU}

Động cơ không đồng bộ sáu pha (SPIM - SixPhase Induction Motor) sở hữu nhiều ưu điểm hơn so với động cơ không đồng bộ ba pha truyền thống. Một số ưu điểm có thể kể đến như giảm xung mômen, tổn hao hài rotor, mất một hay nhiều pha hệ thống vẫn có thể hoạt động ở mức công suất thấp hơn (Williamson \& Smith, 2003), dòng điện trên mỗi pha SPIM và mỗi chân biến tần nguồn áp cũng có giá trị thấp hơn nên không cần phải sử dụng linh kiện bán dẫn với tần số đóng cắt cao, công suất lớn (Golubev \& Ignatenko, 2000). Với những ưu điểm nêu trên thì SPIM thường được ứng dụng vào các hệ thống đòi hỏi công suất lớn, độ tin cậy cao như các ngành ô tô, xe điện, tàu thủy,... (Ojeda et al., 2016).

Có nhiều phương pháp được sử dụng để điều khiển SPIM như: kỹ thuật điều khiển vô hướng V/f, kỹ thuật điều khiển FOC, DTC,...(Finch \& Giaouris, 2008). Trong đó, kỹ thuật điều khiển vô hướng V/f cung cấp chất lượng ở chế độ quá độ thấp, vì vậy được sử dụng trong các ứng dụng không đòi hỏi độ chính xác cao. Ngược lại, trong các hệ truyền động SPIM yêu cầu chất lượng điều khiển cao thì kỹ thuật điều khiển FOC hoặc DTC thường được sử dụng. Tuy nhiên, muốn sử dụng được các kỹ thuật điều khiển nêu trên thì việc đầu tiên và quan trọng nhất là phải ước lượng được thông số của mô hình hay còn được gọi là nhận dạng mô hình. Nhiều nghiên cứu gần đây đã đi sâu vào chủ đề như phân tích hiệu suất của SPIM (Mandal, 2015) và điều khiển SPIM (Bojoi et al., 2005) nhưng chưa có nghiên cứu cụ thể nào về việc nhận dạng thông số, vì vậy trong bài báo này sẽ trình bày cơ sở lý thuyết và mô phỏng bài toán nhận dạng SPIM trên hệ trục cố định $(\alpha \beta)$, tạo ra tiền đề để áp dụng các kỹ thuật điều khiển sau này. Tốc độ quay rotor, mômen xoắn và từ thông rotor là những thông số được tiến hành nhận dạng trong nghiên cứu.

Bài toán nhận dạng cần giải quyết bốn vấn đề sau (Huỳnh Thái Hoàng, 2006): thí nghiệm thu thập dữ liệu (bỏ qua bước này khi sử dụng phương pháp nhận dạng online), chọn cấu trúc mô hình, chọn tiêu chuẩn ước lượng thông số; đánh giá chất lượng mô hình

Ngày nay, các công cụ phổ biến được sử dụng trong bài toán nhận dạng có thể kể đến như: giải thuật di truyền (Huỳnh Thái Hoàng, 2006), mô hình logic mờ (Tanaka \& Tanino, 1994), mạng neuron truyền thẳng và mạng neuron $\mathrm{RBF}(\mathrm{Xie} \& \mathrm{Yu}$, 2011),... mỗi công cụ đều có những đặc thù, ưu điểm và nhược điểm riêng, tuy nhiên khi áp dụng bài toán nhận dạng đối tượng MIMO phi tuyến thì phương pháp mạng neuron RBF cho thấy có nhiều ưu điểm hơn. Một số ưu điểm có thể kể đến như: thời gian đáp ứng nhanh, trọng số tuyến tính ở lớp ra độc lập với các neuron ở lớp ẩn, hoạt động tốt và ổn định khi có nhiễu tác động. Với những ưu điểm nêu trên nhóm tác giả đã lựa chọn phương pháp mạng neuron RBF để nhận dạng các thông số SPIM, đây là hệ thống phi tuyến nhiều ngõ vào và nhiều ngõ ra (MIMO - Multi Input Multi Output). Mạng neuron RBF được sử dụng với những ưu điểm sau:

- Không cần biết thông tin về đối tượng nhận dạng. nhanh.

- Chỉ cần một lớp ẩn và thời gian đáp ứng

- Huấn luyện online,...

\section{MÔ HÌNH TOÁN ĐộNG CƠ KHÔNG ĐỒNG BỘ SÁU PHA}

Xây dựng mô hình toán chính xác cho SPIM là hết sức cần thiết để nghiên cứu và mô phỏng hệ truyền động hoàn chỉnh. Mô hình động cơ có thể được xây dựng theo một trong hai cách sau: thứ nhất, động cơ có thể được biểu diễn bằng hai cặp cuộn dây (hệ trục quay $d q$ ) đại diện cho hai pha ba cuộn dây (Mandal, 2015); thứ hai, dựa trên phân rã không gian vector (hệ trục cố định $(\alpha \beta)$, động cơ có thể được đại diện với hai cặp cuộn dây stator được biểu diễn trên khung tham chiếu stator (Ghosh et al., 2019). Ở cả hai cách tiếp cận, thành phần thứ tự không được bỏ qua vì các điểm trung tính của hai cuộn dây ba pha được cách ly.

Trong bài báo này, mô hình SPIM được xây dựng dựa trên phương pháp phân rã không gian vector (VSD - Vector Space Descriptor). Lý thuyết VSD được dùng để biến đổi không gian sáu chiều ban đầu của SPIM thành ba không gian con trực giao hai chiều trong khung tham chiếu cố định $(\alpha \dot{\beta}),(x y)$ và $(z 1 z 2)$. Từ cách tiếp cận $\mathrm{VSD}$, có thể thấy rằng chuyển đổi năng lượng điện cơ chỉ diễn ra trong không gian con $(\alpha \beta)$, trong khi đó các thành phần dòng trong không gian con $(x y)$ chỉ tạo ra tổn thất. Các vector điện áp trong $(z 1 z 2)$ bằng 0 do cấu hình trung tính cách ly của SPIM. Do đó, hai không gian con $(x y)$ và $(z 1 z 2)$ không ảnh hưởng đến quá trình điều khiển. (Golubev \& Ignatenko, 2000).

Có hai cách phân bố cuộn dây đối với SPIM: Phân bố đối xứng (hai bộ dây ba pha được đặt lệch nhau 60 độ điện); phân bố bất đối xứng (đặt lệch nhau 30 độ điện). Trong đó, SPIM có kiểu phân bố cuộn dây bất đối xứng được ứng dụng rộng rãi hơn. 
Bố trí lệch 30 độ điện giữa hai bộ dây stator giúp SPIM loại bỏ thành phần xung hài mômen bậc sáu cũng như làm giảm đáng kể tổn thất rotor do phân bố này làm giảm các thành phần hài dòng rotor (Jones et al., 2001). Nghiên cứu này trình bày mô hình toán SPIM kiểu rotor lồng sóc. Sơ đồ tương đương với hai bộ dây được đặt lệch 30 độ điện và trung tính cách ly được thể hiện trong (Hình 1). Để xây dựng được mô hình SPIM một số giả thuyết được đưa ra như sau: (Bojoi et al., 2005):

- Khoảng cách khe hở không khí đều, các cuộn dây stator được bố trí đối xứng trong không gian.

- Từ trường và dòng từ hóa được phân bố hình sin trong khe hở không khí.

- Bỏ qua bão hòa từ và tổn hao lỗi thép.

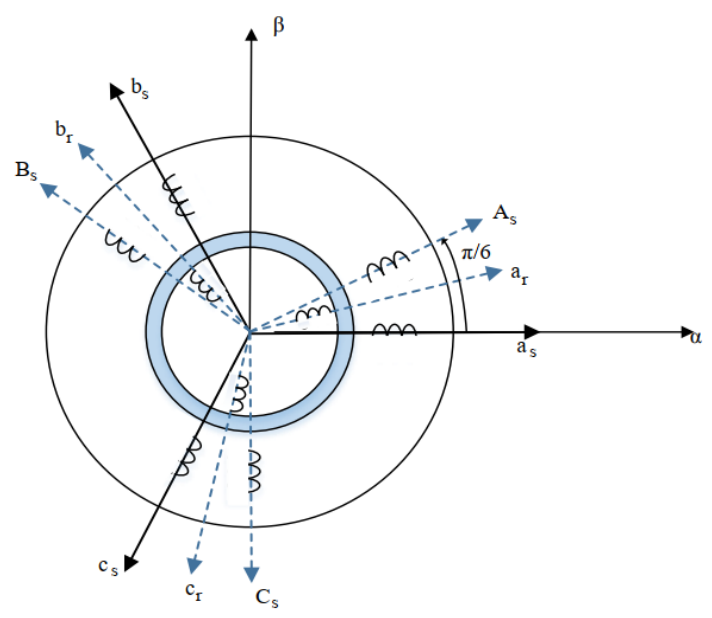

Hình 1. Phân bố các cuộn dây trong SPIM

Trong đó:

$a_{S}, b_{S}, c_{S}$ : Biểu diễn vector bộ dây stator pha $a$, $b, c$ trên hệ trục cố định $(\alpha \beta)$;

$A_{S}, B_{S}, C_{S}$ : Biểu diễn vector bộ dây stator pha $A, B, C$ trên hệ trục cố định $(\alpha \beta)$;

$a_{r}, b_{r}, c_{r}$ : Biểu diễn vector bộ dây rotor pha $a$, $b, c$ trên hệ trục cố định $(\alpha \beta)$;

Bằng cách áp dụng kỹ thuật VSD, không gian sáu chiều ban đầu của SPIM được chuyển thành ba không gian hai chiều trong ba hệ tọa độ cố định $(\alpha \beta),(x y)$ và $(z 1 z 2)$. Sự chuyển đổi này được thực hiện thông qua ma trận chuyển đổi T6 $(6 \times 6)$ (Golubev \& Ignatenko, 2000):

$$
T_{6}=\frac{1}{\sqrt{3}}\left[\begin{array}{cccccc}
1 & -\frac{1}{2} & -\frac{1}{2} & \frac{\sqrt{3}}{2} & -\frac{\sqrt{3}}{2} & 0 \\
0 & \frac{\sqrt{3}}{2} & -\frac{\sqrt{3}}{2} & \frac{1}{2} & \frac{1}{2} & -1 \\
1 & -\frac{1}{2} & -\frac{1}{2} & -\frac{\sqrt{3}}{2} & \frac{\sqrt{3}}{2} & 0 \\
0 & -\frac{\sqrt{3}}{2} & \frac{\sqrt{3}}{2} & \frac{1}{2} & \frac{1}{2} & -1 \\
1 & 1 & 1 & 0 & 0 & 0 \\
0 & 0 & 0 & 1 & 1 & 1
\end{array}\right]
$$

Quá trình biến đổi năng lượng điện cơ chỉ diễn ra trong không gian con $(\alpha \beta)$ và các thành phần hài cơ bản cũng như các thành phần hài bậc $12 n \pm 1(n=1,2,3, \ldots)$ cũng được biểu diễn trong không gian này. Các biến đổi năng lượng điện cơ không được tìm thấy trong hai không gian con $(x y)$ , (z1z2) (Zhao, 1995). Sơ đồ mạch tương đương theo hệ trục cố định $(\alpha \beta)$ được thể hiện trong (Hình 2).

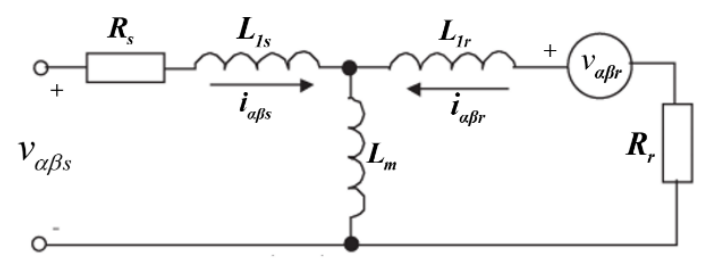

Hình 2. Sơ đồ mạch tương đương theo hệ trục $\alpha \beta$

Phương trình điện áp stator, rotor trên hệ trục $(\alpha \beta)$ (Ghosh et al., 2019):

$$
\begin{aligned}
& v_{\alpha s}=R_{s} i_{\alpha s}+\rho \lambda_{\alpha s}(2.2) \\
& v_{\beta s}=R_{s} i_{\beta s}+\rho \lambda_{\beta s} \\
& v_{\alpha r}=R_{r} i_{\alpha r}+\omega_{r} \lambda_{\beta r}+\rho \lambda_{\alpha r}
\end{aligned}
$$




$$
v_{\beta r}=R_{r} i_{\beta r}-\omega_{r} \lambda_{\alpha r}+\rho \lambda_{\beta r}
$$

Phương trình từ thông stator, rotor trên hệ trục $(\alpha \beta)$ (Ghosh et al., 2019):

$$
\begin{aligned}
& \lambda_{\alpha s}=\left(L_{1 s}+L_{m}\right) i_{\alpha s}+L_{m} i_{\alpha r} \\
& \lambda_{\beta s}=\left(L_{1 s}+L_{m}\right) i_{\beta s}+L_{m} i_{\beta r} \\
& \rho\left[\begin{array}{c}
i_{\alpha s} \\
i_{\beta s} \\
\lambda_{\alpha r} \\
\lambda_{\beta r}
\end{array}\right]=\left[\begin{array}{cc}
\frac{-R_{s}}{\sigma L_{s}}+\frac{R_{r}(1-\sigma)}{\sigma L_{r}} & 0 \\
0 & \frac{-R_{s}}{\sigma L_{s}}+\frac{R_{r}(1-\sigma)}{\sigma L_{r}} \\
L_{m} \frac{R_{r}}{L_{r}} & 0 \\
0 & L_{m} \frac{R_{r}}{L_{r}}
\end{array}\right.
\end{aligned}
$$

Mômen xoắn được trình bày như sau (Ghosh et al., 2019):

$$
T_{e}=\sqrt{\frac{6}{2}} P\left(\left(\lambda_{\beta r}\left(\lambda_{\alpha r}-\frac{L_{m} i_{\alpha s}}{L_{r}}\right)-\lambda_{\alpha r}\left(\lambda_{\beta r}-\frac{L_{m}{ }^{i} \beta s}{L_{r}}\right)\right)\right.
$$

Phương trình tốc độ quay rotor (Ghosh et al., 2019):

$$
\omega_{r}=\frac{1}{J} \int\left(T_{e}-T_{L}\right) d t
$$

Trong đó:

$v_{\alpha s}, v_{\beta s}, v_{\alpha r}, v_{\beta r}$ : Điện áp stator, rotor trên hệ trục $(\alpha \beta)$;

$i_{\alpha s}, i_{\beta s}, i_{\alpha r}, i_{\beta r}$ : Dòng điện stator, rotor trên hệ trục $(\alpha \beta)$;

$\lambda_{\alpha s}, \lambda_{\beta s}, \lambda_{\alpha r}, \lambda_{\beta r}$ : Từ thông stator, rotor trên hệ trục $(\alpha \beta)$;

$\omega_{e}, \omega_{r}$ : Tốc độ điện, tốc độ quay rotor;

$R_{S}, R_{r}$ : Điện trở dây quấn stator, rotor;

$L_{1 s}, L_{1 r}$ : Điện kháng tản của dây quấn stator, rotor;

$L_{s}, L_{r}$ : Điện cảm stator và điện cảm rotor;

$$
\begin{aligned}
& \lambda_{\alpha r}=\left(L_{1 r}+L_{m}\right) i_{\alpha r}+L_{m} i_{\alpha s} \\
& \lambda_{\beta r}=\left(L_{1 r}+L_{m}\right) i_{\beta r}+L_{m} i_{\beta s}
\end{aligned}
$$
2019):

Phương trình không gian trạng thái (Ghosh et al.,

$$
\left.\begin{array}{cc}
\frac{L_{m}}{\sigma L_{s} L_{r}} \frac{R_{r}}{L_{r}} & \frac{L_{m}}{\sigma L_{s} L_{r}} \omega_{r} \\
\frac{L_{m}}{\sigma L_{s} L_{r}} \omega_{r} & \frac{L_{m}}{\sigma L_{s} L_{r}} \frac{R_{r}}{L_{r}} \\
\frac{R_{r}}{L_{r}} & -\omega_{r} \\
\omega_{r} & \frac{R_{r}}{L_{r}}
\end{array}\right]\left[\begin{array}{c}
i_{\alpha s} \\
i_{\beta s} \\
\lambda_{\alpha r} \\
\lambda_{\beta r}
\end{array}\right]+\frac{1}{\sigma L_{s}}\left[\begin{array}{c}
v_{\alpha s} \\
v_{\beta s} \\
0 \\
0
\end{array}\right]
$$

$L_{m}:$ Hỗ cảm giữa stator và rotor;

$T_{e}, T_{L}$ : Mômen xoắn, mômen tải;

$J$ : Mômen quán tính cơ;

$P$ : Số đôi cực;

$$
\begin{aligned}
& \sigma=1-\frac{L_{m}^{2}}{L_{S} L_{r}}: \text { Hệ số từ tản tổng; } \\
& \rho=\frac{d}{d t}
\end{aligned}
$$

\section{MANG NEURON HÀM CƠ SỞ XUYÊN TẦM RBF}

Ngày nay, mạng neuron nhân tạo đang được sử dụng phổ biến trong các giải thuật điều khiển thông minh với nhiều giải thuật khác nhau. Mạng neuron RBF là một trường hợp đặc biệt của mạng neuron truyền thẳng nhiều lớp, mạng có khả năng xấp xỉ hàm rất hiệu quả, ngoài ra mạng $\mathrm{RBF}$ có thể huấn luyện dễ dàng và nhanh chóng. Cấu trúc cơ bản của mạng gồm có một lớp vào, một lớp ẩn và một lớp ra (Liu, 2013).

Mạng neuron RBF được sử dụng trong nghiên cứu này có cấu trúc gồm 8 ngõ vào, 17 neuron ở lớp ẩn và 1 ngõ ra như (Hình 3 ). Các neuron ở lớp ẩn được kích hoạt bằng hàm Gauss. Mỗi neuron lớp ẩn chứa một vector tâm $\mathbf{c}$ có cùng kích thước với vector 
ngõ vào $\mathbf{x}$ được định nghĩa như sau (Liu, 2013): $\left\|x(t)-c_{j}(t)\right\|$

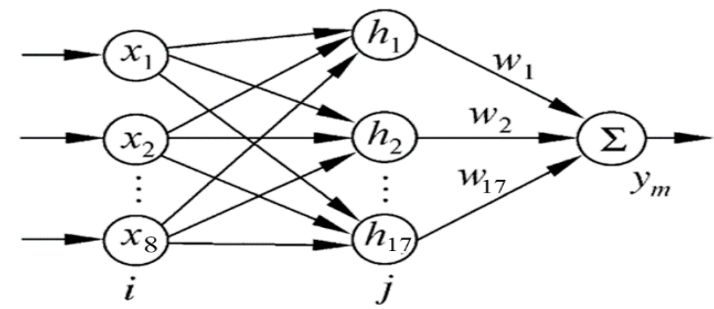

Hình 3. Cấu trúc mạng neuron RBF

Vector ngõ vào $\mathbf{x}$ có dạng:

$$
\boldsymbol{x}=\left[x_{1} x_{2} \ldots x_{8}\right]^{T}
$$

Phương trình hàm Gauss neuron thứ $j$ (Liu, 2013):

$$
\boldsymbol{h}_{\boldsymbol{j}}=\exp \left(-\frac{\left\|\boldsymbol{x}-\boldsymbol{c}_{\boldsymbol{j}}\right\|^{2}}{2 b_{j}^{2}}\right), \text { với } \mathrm{j}=1,2, \ldots, 17(3.2)
$$

Ngõ ra của mạng neuron RBF:

$$
\hat{y}(t)=w_{1} h_{1}+w_{2} h_{2}+\ldots+w_{17} h_{17}
$$

Trong đó:

$c_{\boldsymbol{j}}=\left[c_{j 1} c_{j 2} \ldots c_{j 8}\right]:$ Vector tâm của neuron thứ j;

$\boldsymbol{b}=\left[b_{1} b_{2} \ldots b_{17}\right]^{T}:$ Vector độ rộng của hàm Gauss, với $b_{j}>0$ mô tả giá trị độ rộng của hàm Gauss của neuron thứ $j$;

$$
\boldsymbol{w}=\left[w_{1} w_{2} \ldots w_{17}\right]^{T}: \text { Giá trị trọng số; }
$$

Để huấn luyện mạng neuron RBF, hàm mục tiêu được định nghĩa như sau (Liu, 2013):

$$
E(t)=\frac{1}{2}(y(t)-\hat{y}(t))^{2}
$$

Phương pháp Gradient Descent được áp dụng để cập nhật thông số (Liu, 2013):

$$
\begin{aligned}
& \Delta w_{j}(t)=-\eta \frac{\partial E}{\partial w_{j}}=\eta(y(t)-\hat{y}(t)) h_{j} \\
& w_{j}(t)=w_{j}(t-1)+\Delta w_{j}(t)+\alpha\left[w_{j}(t-1)-w_{j}(t-2)\right] \\
& \Delta b_{j}(t)=-\eta \frac{\partial E}{\partial b_{j}}=\eta[y(t)-\hat{y}(t)] w_{j} h_{j} \frac{\left\|x-c_{j}\right\|^{2}}{b_{j}^{3}} \\
& b_{j}(t)=b_{j}(t-1)+\Delta b_{j}(t)+\alpha\left[b_{j}(t-1)-b_{j}(t-2)\right] \\
& \Delta c_{j i}(t)=-\eta \frac{\partial E}{\partial c_{j i}}=\eta[y(t)-\hat{y}(t)] w_{j} \frac{x_{j}-c_{j i}}{b_{j}^{2}} \\
& c_{j i}(t)=c_{j i}(t-1)+\Delta c_{j i}(t)+\alpha\left[c_{j i}(t-1)-c_{j i}(t-2)\right]
\end{aligned}
$$

Trong đó:

$\eta \in(0,1)$ : Tốc độ học;

$\alpha \in(0,1)$ : Hệ số mômen;

Trọng số ban đầu của các neuron trong lớp ẩn có thể được chọn dựa vào sự phân nhóm dữ liệu vào. Quá trình huấn luyện chủ yếu chỉnh định trọng số tuyến tính ở lớp ra. Các trọng số tuyến tính ở lớp ra có thể xem như độc lập với các neuron ở lớp ẩn. Khả năng xấp xỉ chính xác và tốc độ của mạng $R B F$ còn có thể cải thiện hơn nữa bằng việc chọn tâm, độ rộng phù hợp cho các hàm cơ sở ở lớp ẩn. Tuy nhiên, mạng $\mathrm{RBF}$ thường đáp ứng chậm trong giai đoạn huấn luyện nếu số lượng neuron trong lớp ẩn được chọn quá lớn.

Phần tiếp theo sẽ ứng dụng mạng RBF để nhận dạng tốc độ quay, mômen xoắn, từ thông rotor SPIM và kết quả mô phỏng đạt được.

\section{NHẬN DẠNG SPIM TRÊN HÊ TRỤC ( $\alpha \beta)$ SỦ DỤNG MẠNG NEURON RBF}

\subsection{Sơ đồ mô phỏng}

Sơ đồ mô phỏng hệ thống nhận dạng SPIM sử dụng mạng neuron RBF trên phần mềm Matlab/Simulink có dạng như sau (Hình 4): 


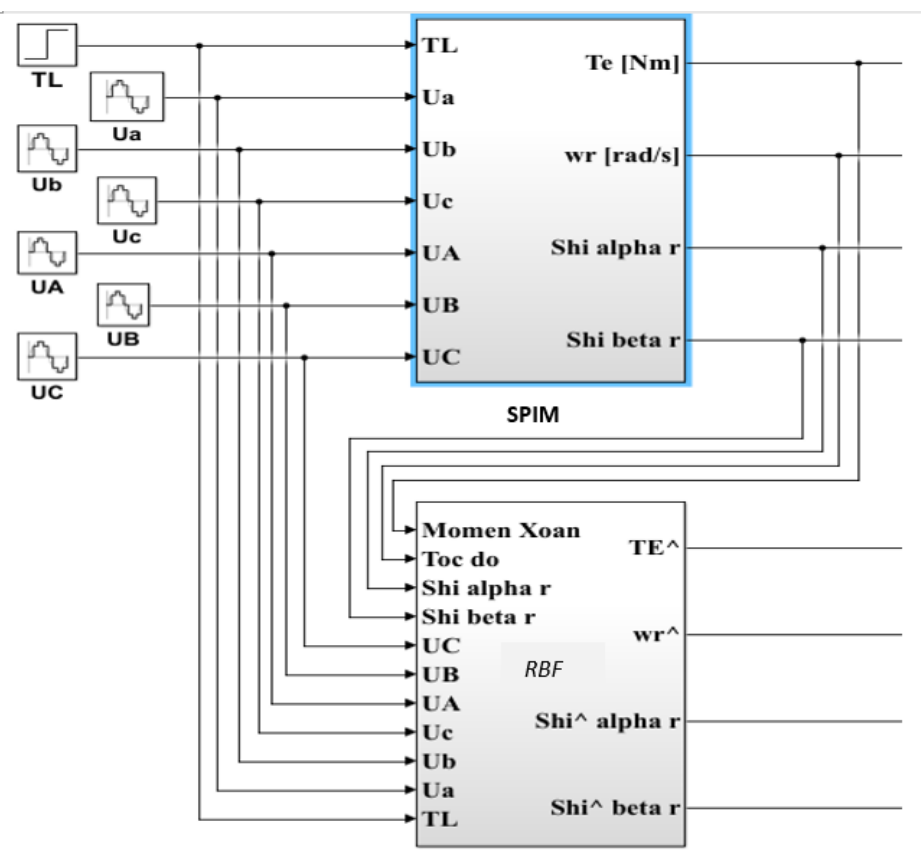

Hình 4. Sơ đồ hệ thống nhận dạng SPIM tải.

- Ngõ vào đối tượng: Điện áp sáu pha, mômen

- Ngõ ra đối tượng: Mômen xoắn, tốc độ quay và từ thông rotor.

- Ngõ vào bộ nhận dạng RBF: Điện áp sáu pha, mômen tải, mômen xoắn, tốc độ quay và từ thông rotor.

- Ngõ ra bộ nhận dạng RBF: Mômen xoắn, tốc độ quay và từ thông rotor.

Kết quả mô phỏng bộ nhận dạng mômen xoắn, tốc độ quay và từ thông rotor trên hệ trục $(\alpha \beta)$ được trình bày ở phần tiếp theo.

\subsection{Kết quả mô phỏng}

Thông số của đối tượng được tham khảo từ tài liệu (Mandal, 2015):

$$
L_{1 s}=0,0132 H ; L_{m}=1 H ; L_{1 r}=0,0132 H ;
$$

$R_{S}=1,9 \Omega ; R_{r}=2,1 \Omega ; V_{\text {an }}=230 \mathrm{~V} ; p=2 ;$

$\omega=314 \mathrm{rad} / \mathrm{s} ; J_{r}=0,02 \mathrm{~kg} \cdot \mathrm{m}^{2}$;

Thông số mạng neuron RBF được khởi tạo như sau:

$$
\alpha=0,9 ;
$$

Tâm $c$ được chọn với giá trị (Ma trận $8 \times 17$, hàng 2 đến hàng 8 có giá trị giống nhau):

$$
\left[\begin{array}{ccccccccccccccccc}
-10 & -8,75 & -7,5 & -6,25 & -5 & -3,75 & -2,5 & -1,25 & 0 & 1,25 & 2,5 & 3,75 & 5 & 6,25 & 7,5 & 8,75 & 10 \\
-1 & -0.875 & -0.75 & -0.625 & -0.5 & -0.375 & -0.25 & -0.125 & 0 & 0.125 & 0.25 & 0.375 & 0.5 & 0.625 & 0.75 & 0.875 & 1 \\
& & & & & & & & \vdots & & & & & & & & \\
-1 & -0.875 & -0.75 & -0.625 & -0.5 & -0.375 & -0.25 & -0.125 & 0 & 0.125 & 0.25 & 0.375 & 0.5 & 0.625 & 0.75 & 0.875 & 1
\end{array}\right]
$$

$\boldsymbol{b}=[200 ; 200 ; 200 ; 200 ; 200 ; 200 ; 200 ; 200 ; 200 ; 200 ; 200 ; 200 ; 200 ; 200 ; 200 ; 200 ; 200]$;

$$
\boldsymbol{w}=[0,23 ; 0,15 ; 0,06 ;-0,44 ;-0,50 ;-0,09 ;-0,54 ; 0,60 ; 0,97 ;-0,94 ; 0,07 ;-0,82 ; 0,60 ; 0,97 ;-0,86 ; 0,87 ;-0,96] \text {; }
$$

Kết quả bộ nhận dạng và sai số nhận dạng tín hiệu mômen xoắn được thể hiện trên hình (Hình 5 , Hình 6), tốc độ quay (Hình 7, Hình 8) và từ thông rotor trên hệ trục $(\alpha \beta)$ (Hình 9, Hình 10, Hình 11 và Hình 12). Trong đó (Hình 5 , Hình 7 , Hình 9 và Hình 11) thể hiện cùng lúc đáp ứng của đối tượng và của bộ nhận dạng để dễ dàng so sánh. Nét liền thể hiện đáp ứng đối tượng và nét đứt đậm thể hiện đáp ứng bộ nhận dạng. 


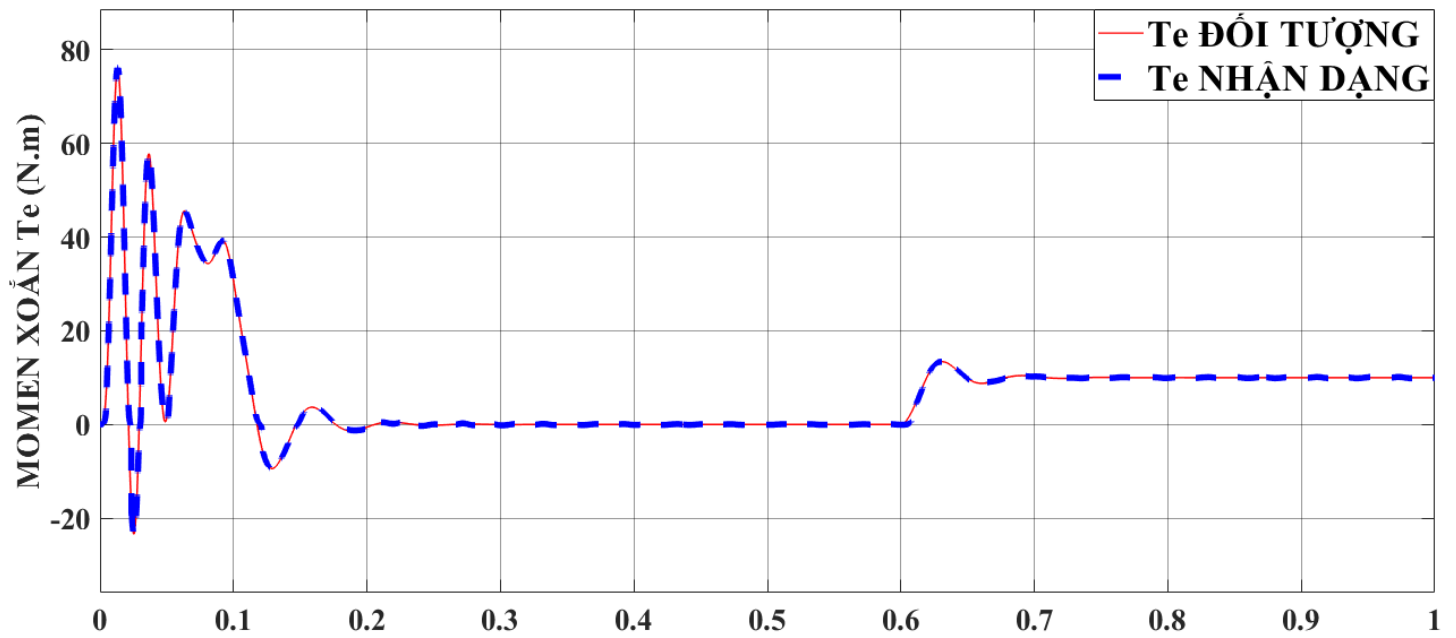

Hình 5. Kết quả nhận dạng mômen xoắn. Đáp ứng của đối tượng và bộ nhận dạng được thể hiện trên cùng một hình

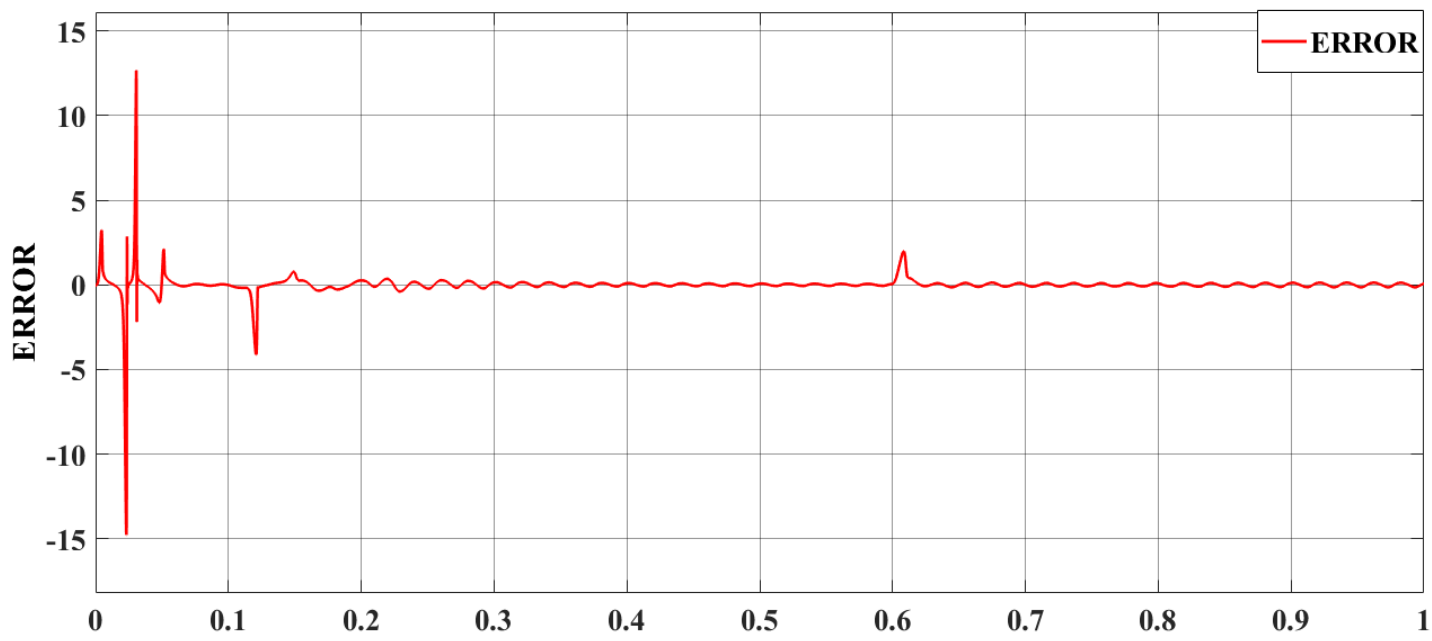

Hình 6. Sai số mômen xoắn

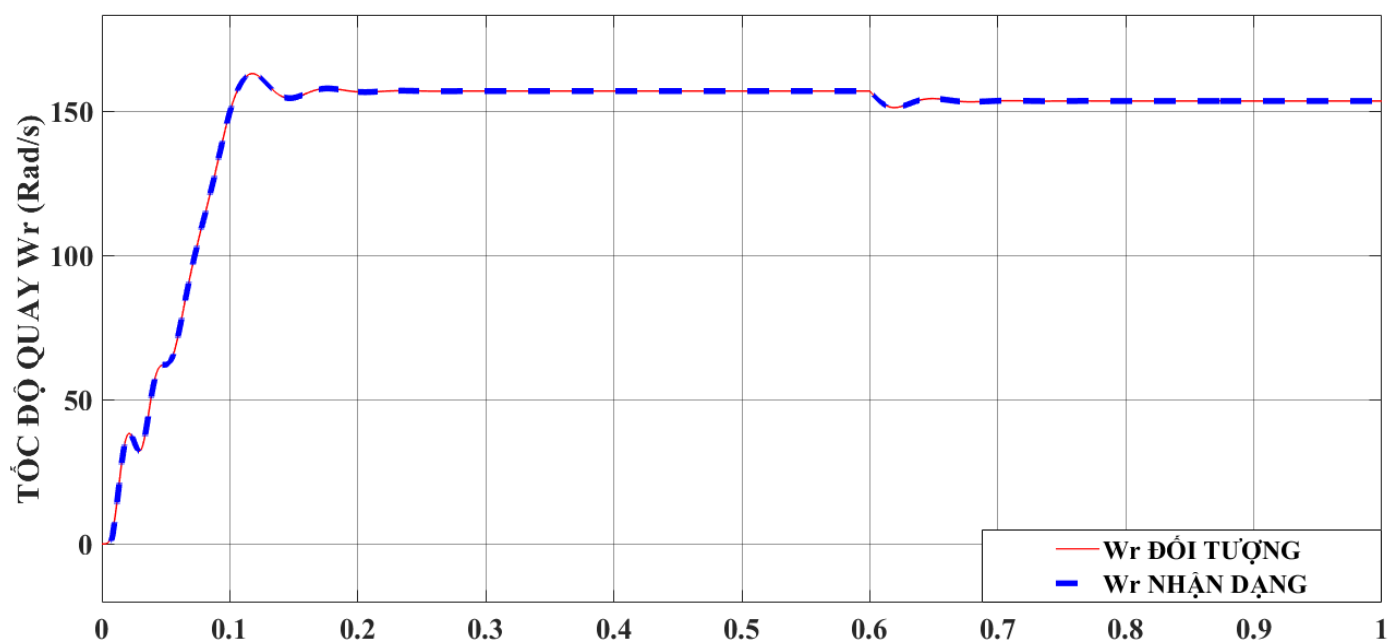

Hình 7. Kết quả nhận dạng tốc độ quay rotor 


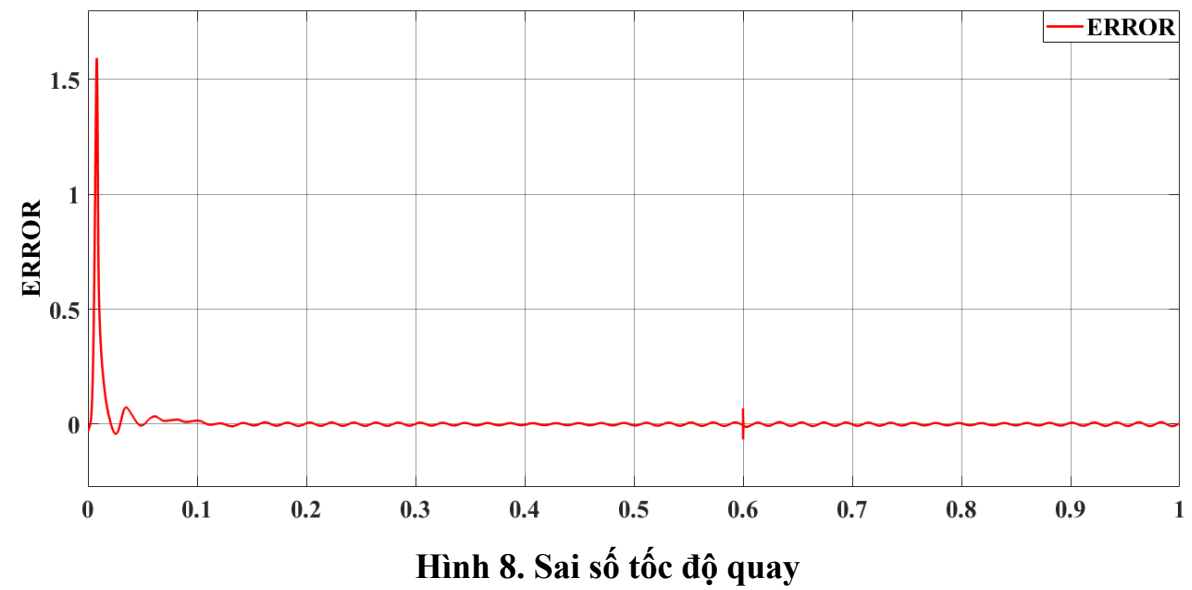

Quan sát các kết quả mô phỏng về bộ nhận dạng mômen xoắn và tốc độ quay SPIM được thể hiện ở hình (Hình 5 , Hình 6 , Hình 7 , Hình 8 ) cho thấy rằng đáp ứng của bộ nhận dạng bám theo đáp ứng của đối tượng:

- Trong (Hình 6) sau khoảng 0,2 giây sai số mômen xoắn được xác lập. Tải được lắp vào động cơ tại thời điểm 0,6 giây nên làm cho sai số mômen xoắn có độ vọt lố là 2 và bị dao động trong khoảng
0,02 giây. Sau khoảng thời gian 0,02 giây thì sai số vẫn tiếp tục hội tụ về 0 .

- Sai số tốc độ quay được thể hiện ở (Hình 8), đáp ứng xác lập trong khoảng 0,1 giây. Thời điểm 0,6 giây tải được lắp vào động cơ nên đáp ứng có độ vọt lố nhưng không đáng kể.

- Độ phù hợp giữa đáp ứng ngõ ra và đáp ứng nhận dạng mômen xoắn có giá trị xấp xỉ $99,8 \%$ và tốc độ quay rotor là 99,95\%. Độ phù hợp được tính dựa trên (Nguyễn Hoàng Dũng, 2013).

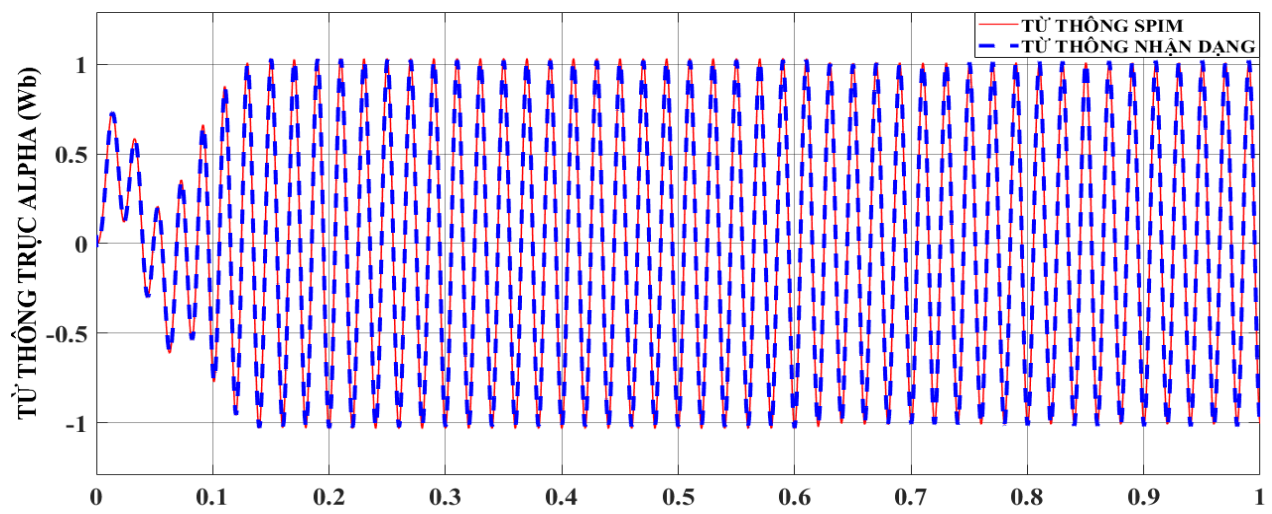

Hình 9. Kết quả nhận dạng từ thông rotor trên trục $\alpha$

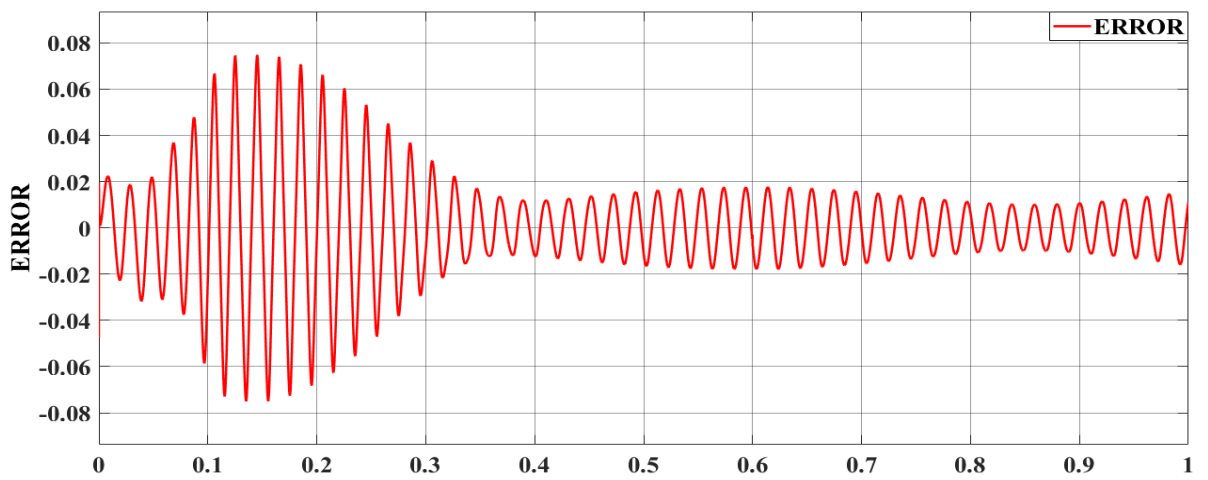

Hình 10. Sai số từ thông rotor trên trục $\alpha$ 


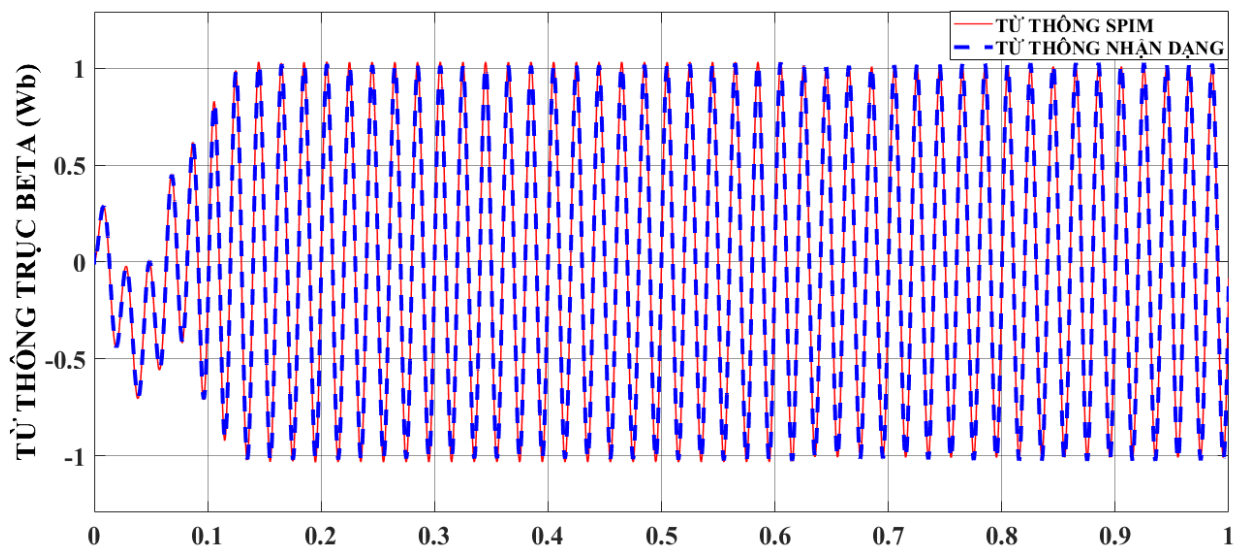

Hình 11. Kết quả nhận dạng từ thông rotor trên trục $\beta$

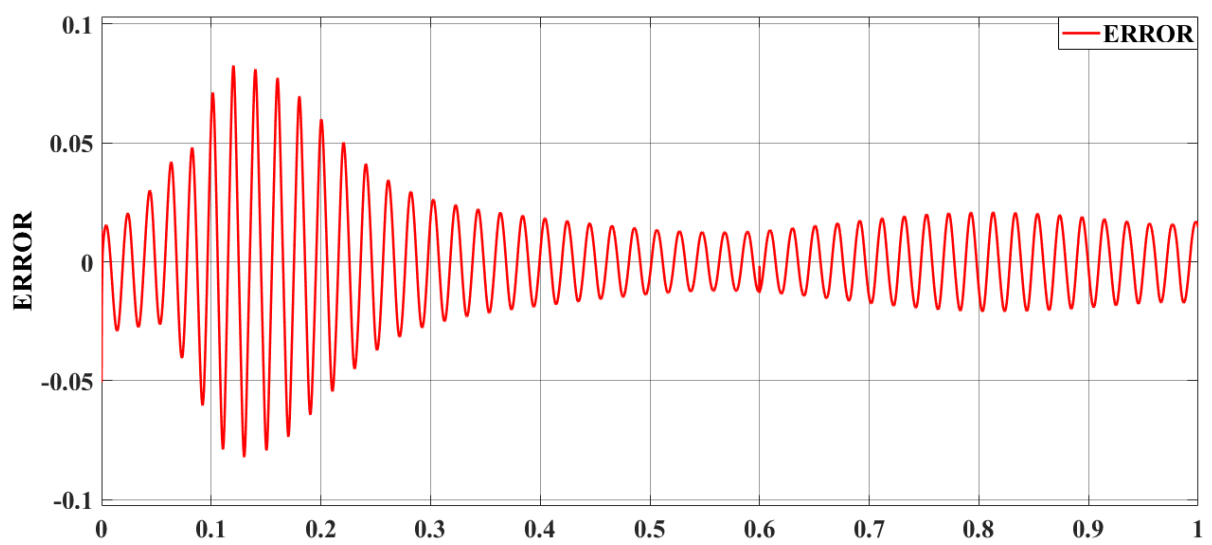

Hình 12. Sai số từ thông rotor trên trục $\beta$

Kết quả mô phỏng về bộ nhận dạng từ thông rotor trên hệ trục cố định $(\alpha \beta)$ được thể hiện trong (Hình 9, Hình 10, Hình 11 và Hình 12). Kết quả cho thấy từ thông bộ nhận dạng bám theo từ thông của đối tượng sau khoảng thời gian 0,4 giây.

Thời điểm 0,4 giây động cơ hoàn tất quá trình khởi động, đáp ứng ở (Hình 10 và Hình 12) cho thấy bộ nhận dạng vẫn hoạt động ổn định, sai số nhận dạng thấp.

Độ phù hợp giữa đáp ứng ngõ ra và đáp ứng nhận dạng từ thông rotor trên trục $\alpha$ có giá trị xấp xỉ $99,82 \%$ và trên trục $\beta$ là $99,85 \%$.

Khảo sát trường hợp hệ thống có nhiễu trắng tác động với công suất 0,001 (Hình 13):

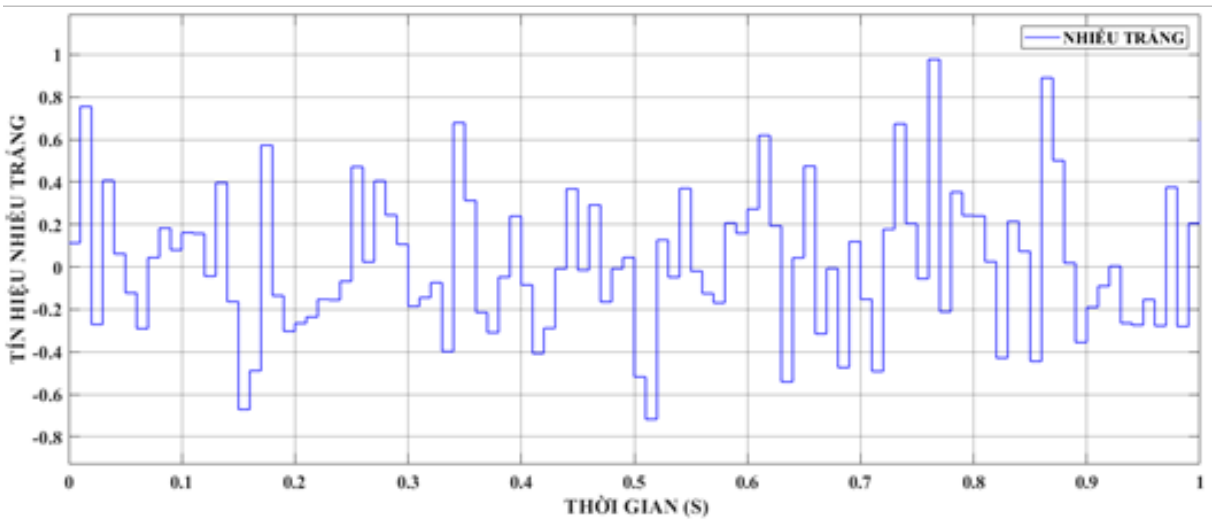

Hình 13. Tín hiệu nhiễu trắng 


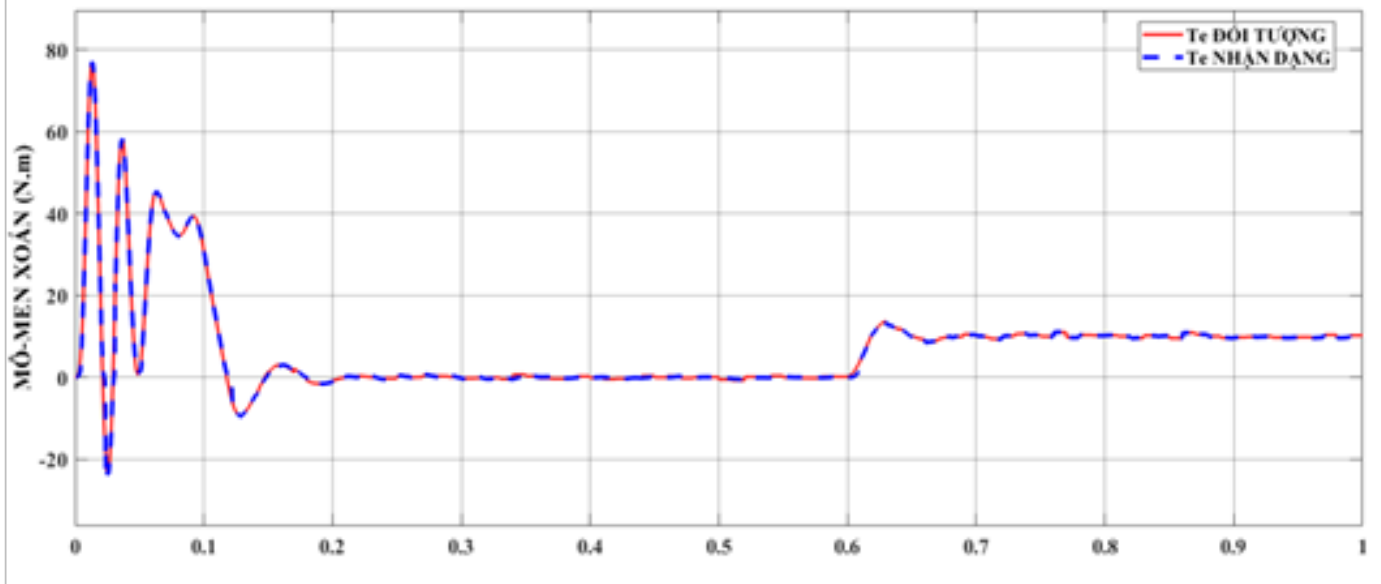

Hình 14. Kết quả nhận dạng mômen xoắn trường hợp có nhiễu tác động

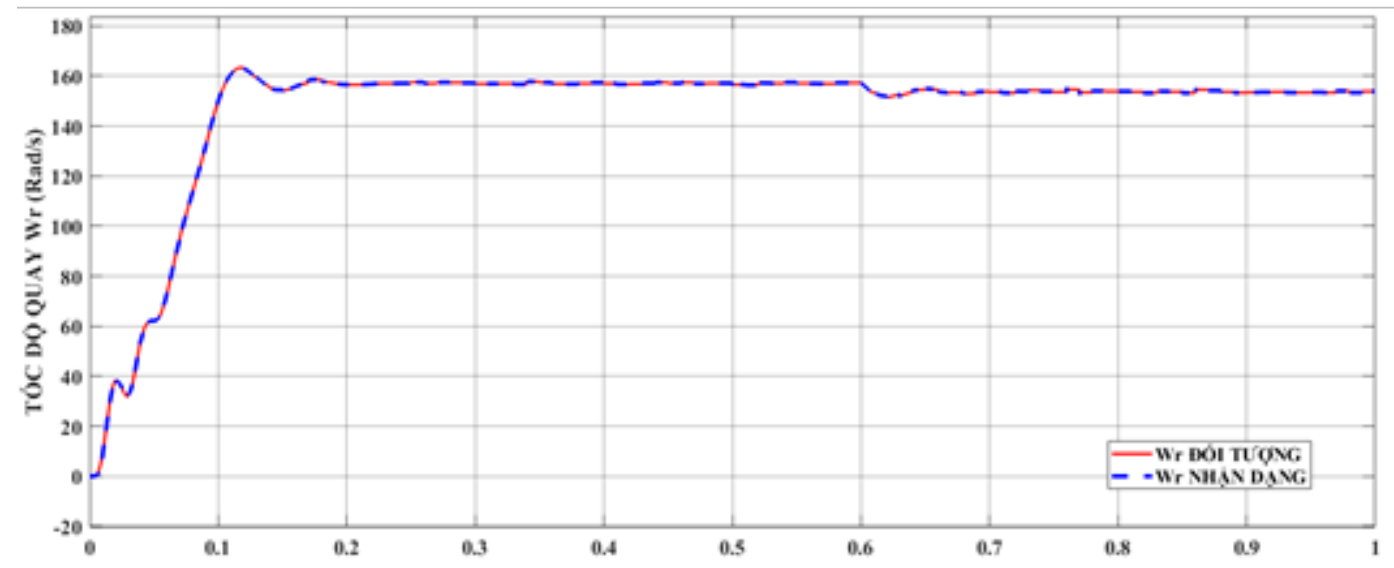

Hình 15. Kết quả nhận dạng tốc độ quay rotor trường hợp có nhiễu tác động

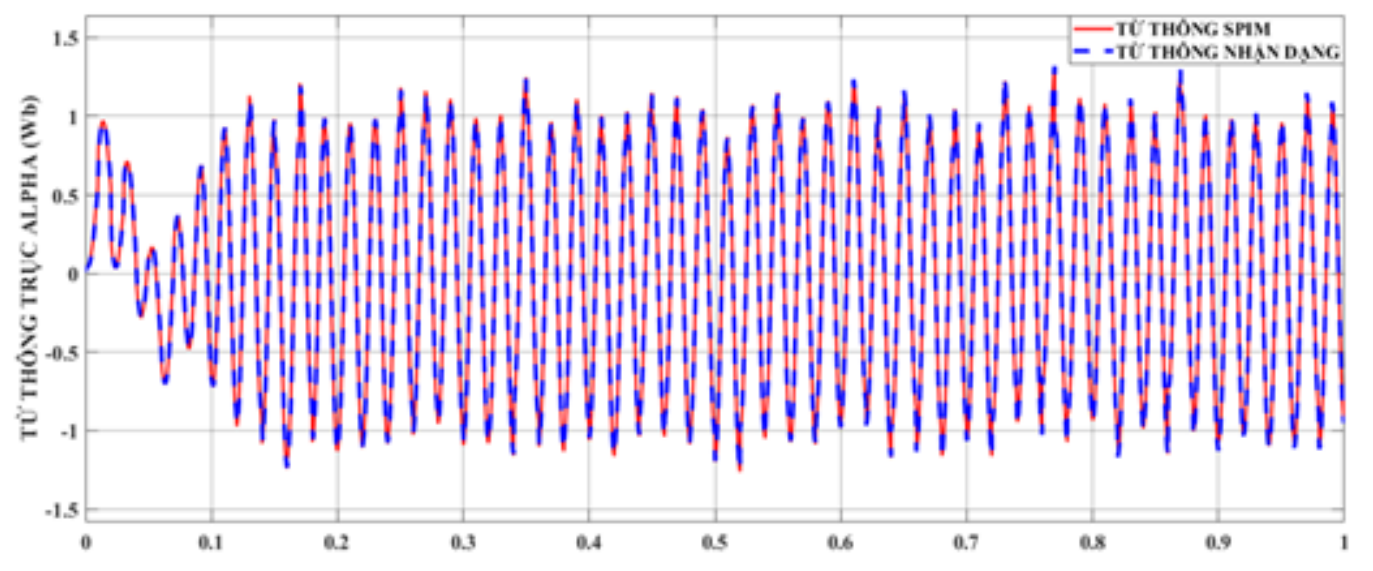

Hình 16. Kết quả nhận dạng từ thông rotor trục $\alpha$ trường hợp có nhiễu tác động 


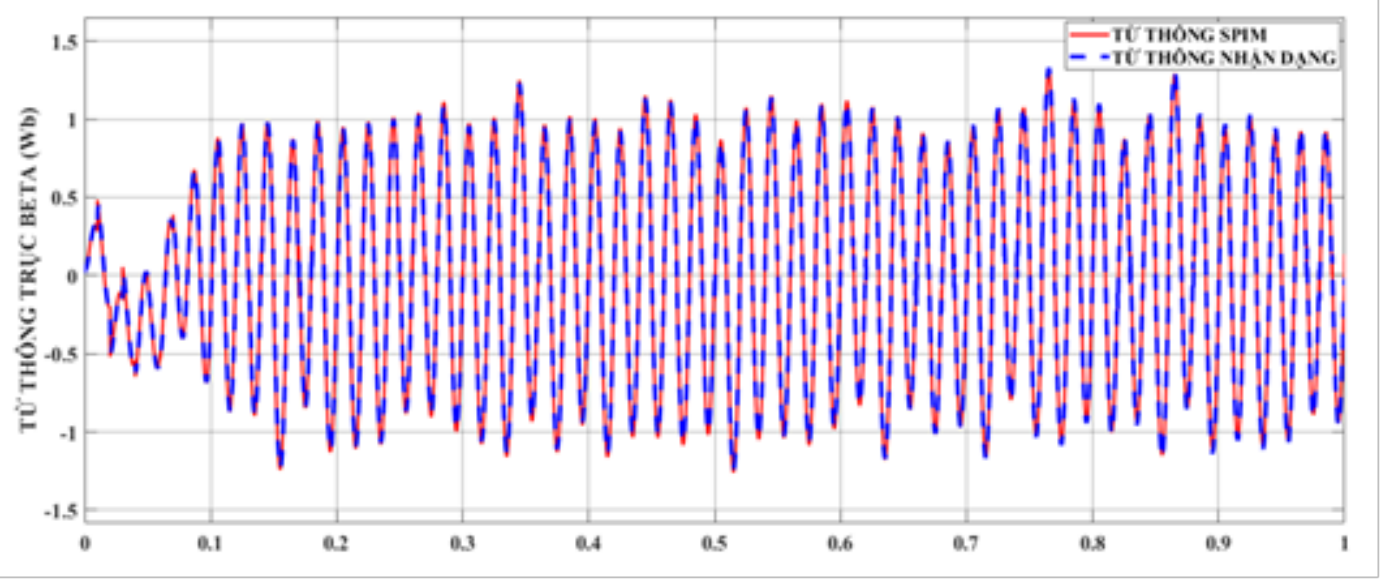

Hình 17. Kết quả nhận dạng từ thông rotor trục $\beta$ trường hợp có nhiễu tác động

Kết quả mô phỏng về bộ nhận dạng mômen xoắn, tốc độ quay rotor và từ thông rotor trên hệ trục cố định $(\alpha \beta)$ trong trường hợp có nhiễu trắng tác động được thể hiện trong (Hình 14, Hình 15, Hình 16 và Hình 17). Kết quả cho thấy đáp ứng của bộ nhận dạng vẫn hoạt động tốt và không bị mất ổn định. Độ phù hợp giữa đáp ứng ngõ ra và đáp ứng nhận dạng có giá trị lần lượt như sau: Mômen xoắn xấp xỉ 99,21\%, tốc độ quay rotor xấp xỉ 99,38\%, từ thông rotor trên trục $\alpha$ xấp xỉ $98,85 \%$ và trên trục $\beta$ là $98,89 \%$.

Việc áp dụng luật cập nhật trọng số online để nhận dạng đối tượng bằng mạng neuron RBF thông qua mô phỏng trên phần mềm Matlab/Simulink cho thấy được ưu điểm của thuật toán:

- Đáp ứng từ thông trên hệ trục $(\alpha \beta)$ của bộ nhận dạng luôn bám sát từ thông của đối tượng sau thời gian khoảng 0,4 giây.

- Sai số nhận dạng tiến về 0 . Hệ thống làm việc xác lập và ổn định trong trường hợp động cơ khởi động và sau khi lắp tải.

\section{KẾT LUẬN}

Bài báo đã trình bày phương pháp nhận dạng SPIM, cụ thể là nhận dạng các thông số mômen xoắn, tốc độ quay và từ thông rotor trên hệ trục cố định $(\alpha \beta)$ sử dụng phương pháp mạng neuron RBF. Kết quả mô phỏng sử dụng phần mềm Matlab/Simulink cho thấy bộ nhận dạng đạt hiệu quả cao, sai số nhận dạng hội tụ về 0 và độ phù hợp giữa đáp ứng ngõ ra và đáp ứng nhận dạng nằm trong khoảng 98\%-99\% khi hệ thống hoạt động không tải, có tải và nhiễu trắng tác động. Nghiên cứu này là tiền đề để việc điều khiển SPIM khi sử dụng phương pháp truyền thống hay thông minh như
FOC, DTC, Logic mờ,... được thuận lợi hơn vì có thể ước lượng được giá trị tức thời mômen xoắn, tốc độ quay và từ thông rotor. Nhóm tác giả sẽ tiếp tục nghiên cứu giải thuật tối ưu để lựa chọn được số lượng neuron ở lớp ẩn và thông số của mạng được ít nhất và tối ưu nhất vì hiện tại số lượng neuron và thông số của mạng còn được lựa chọn và khởi tạo bằng kinh nghiệm.

\section{TÀI LIẸU THAM KHẢO}

Bojoi, R., Farina, F., Griva, G. \& Profumo, F. (2005). Direct Torque Control for Dual Three Phase Induction Motor Drives. IEEE Transactions on Industry Applications, 41(6), 1627-1635.

Finch, J. \& Giaouris, D. (2008). Controlled AC electrical drives. IEEE Trans. Ind. Electron, 39(4), 986-993.

Ghosh, B. C., Habibullah, M., Ali, M. E. (2019). Performance Comparison of Five and Six-Phase Induction Motors Operating under Normal and Faulty Conditions. Electrical Information and Communication Technology (pp. 140-146).

Golubev, A.N., \& Ignatenki, S.V. (2000). Influence of number of stator-winding phases on the noise characteristics of an asynchronous motor. Russ. Electr. Eng., 71(6), 41-46.

Huỳnh Thái Hoàng. (2006). Hệ thống điều khiển thông minh. NXB Đại học Quốc gia TP. Hồ Chí Minh.

Jones, M., Vukosavic, S. N., \& Levi, E. (2001). Experimental performance evaluation of sixphase series - connected two - motor drive systems. European Power Electronics and Applications Conf. EPE (pp. 95-102).

Liu, J. (2013). Radial Basis Function (RBF) neural network control for mechanical systems: Design, analysis and Matlab simulation. Springer Science $\&$ Business Media. 
Mandal, M. S. (2015). Performance Analysis of SixPhase Induction Motor. IJERT, 60(4), 589-593.

Nguyễn Hoàng Dũng. (2013). Đồng bộ hệ CHAOTIC LORENZ dùng bộ điều khiển trượt. Tạp chí Khoa hoc Trưòng Đại hoc Cần Tho, 27, 25-30.

Ojeda, J.., Bouker, H., Vido, L. \& Ben Ahmed, H. (2016). Comparison of 3-phase and 5-phase high speed synchronous motor for $\mathrm{EV} / \mathrm{HEV}$ applications. IEEE Industry Applications Society Ann. Meeting IAS, 60(6), 250-265.
Tanaka, M., \& Tanino, T. (1994). Identification of Nonlinear Systems using Fuzzy Logic and Genetic Algorithms. IFAC Syst. Identif., 50(4), 265-270.

Williamson, S., \& Smith, S. (2003). Pulsating torque and losses in multiphase induction machines. IEEE Trans. Ind. Appl, 39(4), 986-993.

Zhao, Y. (1995). Space Vector PWM Control of Dual Threephase Induction Machine using Vector Space Decomposition. IEEE Trans. Ind. Appl, 56(2), 1100-1108. 